\title{
Biofabrication of customized bone grafts by combination of additive manufacturing and bioreactor knowhow
}

\author{
Pedro F Costa ${ }^{1,2}$, Cédryck Vaquette ${ }^{3}$, Jeremy Baldwin ${ }^{3}$, Mohit Chhaya ${ }^{3}$, \\ Manuela E Gomes ${ }^{1,2}$, Rui L Reis ${ }^{1,2}$, Christina Theodoropoulos ${ }^{3}$ \\ and Dietmar W Hutmacher ${ }^{3,4}$ \\ 1 3B's Research Group-Biomaterials, Biodegradables and Biomimetics, University of Minho, \\ Headquarters of the European Institute of Excellence on Tissue Engineering and Regenerative Medicine, \\ Guimarães, Portugal \\ 2 ICVS/3B's-PT Government Associate Laboratory, Braga, Portugal \\ ${ }^{3}$ Institute of Health and Biomedical Innovation, Queensland University of Technology, Brisbane, \\ Australia \\ ${ }^{4}$ Institute for Advanced Studies, Technical University of Munich, Munich, Germany \\ E-mail: dietmar.hutmacher@qut.edu.au
}

Received 7 September 2013, revised 13 March 2014

Accepted for publication 28 March 2014

Published 8 May 2014

\begin{abstract}
This study reports on an original concept of additive manufacturing for the fabrication of tissue engineered constructs (TEC), offering the possibility of concomitantly manufacturing a customized scaffold and a bioreactor chamber to any size and shape. As a proof of concept towards the development of anatomically relevant TECs, this concept was utilized for the design and fabrication of a highly porous sheep tibia scaffold around which a bioreactor chamber of similar shape was simultaneously built. The morphology of the bioreactor/scaffold device was investigated by micro-computed tomography and scanning electron microscopy confirming the porous architecture of the sheep tibiae as opposed to the non-porous nature of the bioreactor chamber. Additionally, this study demonstrates that both the shape, as well as the inner architecture of the device can significantly impact the perfusion of fluid within the scaffold architecture. Indeed, fluid flow modelling revealed that this was of significant importance for controlling the nutrition flow pattern within the scaffold and the bioreactor chamber, avoiding the formation of stagnant flow regions detrimental for in vitro tissue development. The bioreactor/scaffold device was dynamically seeded with human primary osteoblasts and cultured under bi-directional perfusion for two and six weeks. Primary human osteoblasts were observed homogenously distributed throughout the scaffold, and were viable for the six week culture period. This work demonstrates a novel application for additive manufacturing in the development of scaffolds and bioreactors. Given the intrinsic flexibility of the additive manufacturing technology platform developed, more complex culture systems can be fabricated which would contribute to the advances in customized and patient-specific tissue engineering strategies for a wide range of applications.
\end{abstract}

Keywords: additive manufacturing, bioreactor, tissue engineering, biofabrication, custom-made scaffolds

S Online supplementary data available from stacks.iop.org/BF/6/035006/mmedia

(Some figures may appear in colour only in the online journal) 


\section{Introduction}

Personalized medical therapy is an emerging practice offering tailored solutions to each individual. This approach is envisioned to revolutionize healthcare, through costeffectiveness, efficiency and improved patient outcomes. Currently, the pharmaceutical industry is investing heavily in this approach through the development of personalized medicine supported by the advances in molecular and genomic technologies [1]. To some extent, the field of tissue engineering and regenerative medicine is following a similar path with increasing attention given to the development of customized tissue engineered products that would meet the patient-specific requirements for repair and/or regeneration of tissues [2].

Additive manufacturing (AM) is one of the many engineering technologies that have been utilized in tissue engineering and it is central for the fabrication of customized implants. AM builds objects layer-by-layer by gradual deposition of materials in an organized fashion, permitting the fabrication of three-dimensional (3D) objects with highly complex architecture [3]. However, the development of customized constructs of large dimensions and their subsequent cell culture have remained challenging because of the requirements for appropriate oxygen and nutrient diffusion throughout the entire construct. By utilizing bioreactors, rather than traditional static cultures, enhanced fluid diffusion within the scaffold can be achieved, permitting in vitro culture under highly controlled conditions [4]. Bioreactors are usually designed to meet specific requirements for a standardized scaffold size and shape, type of perfusion, desired fluid flow pattern, type of applied stimulatory factors (biochemical or biomechanical) and integrated sensorial probes [5-11]. These points exemplify the degree of complexity achieved in traditional bioreactors as well as the lack of versatility when anatomical scaffolds are considered for tissue engineering applications. Indeed, this represents a major limitation to the generalized use of bioreactors in personalized tissue engineering strategies as any modifications of a standardized bioreactor chamber engender considerable additional engineering steps and therefore result in significantly increased labour and cost.

Control over the fluid flow pattern within the construct is a crucial parameter for the proliferation and survival of cultured cells in 3D. Shear stress resulting from fluid perfusion is known to influence cell behaviour. More importantly, perfusion is able to increase cell survival and hence culture efficiency by avoiding the formation of regions where the culture medium is stagnant as well as regions of high fluid intensities capable of detaching the cells from their substrates [12-18]. This suggests that the architecture of the bioreactor should be similar to that of the scaffold in order to achieve homogeneous fluid perfusion and alleviate the aforementioned issues. A significant advance towards the development of anatomically shaped bioreactor chambers was performed by Grayson et al [11] who fabricated a scaffold resembling a bovine temporomandibular joint (TMJ) condylar bone and cultured the resulting construct in a bioreactor of similar dimensions. The anatomically shaped bioreactor's chamber was produced by casting polydimethylsiloxane around a TMJlike mould, inserted into a cylindrical tube which was utilized to hold the scaffold for achieving appropriate fluid transfer to the construct's most central portions. This technique required multiple steps and lacked versatility for facilitating the addition of new bioreactor features such as additional inlets and variation in the scaffold dimensions. Although promising, this approach still requires revision of the bioreactor design when tailoring to suit anatomically different samples, indicating that even though scaffolds can easily be fabricated into an array of unique but specific anatomical dimensions, it is not necessarily the case for bioreactor chambers.

Based on this background, we hypothesized that additive manufacturing could be utilized to simultaneously fabricate both the scaffold and the bioreactor chamber and provide sufficient flexibility for rapidly manufacturing devices with various anatomical shapes. This concept inherits a high degree of process automation, hence enabling the fabrication of high-quality personalized tissue engineered constructs in a reproducible manner and at an efficient cost and speed. This study reports on a proof of concept that additive manufacturing involving a commercially inexpensive dual polymer extrusion system is capable of fabricating anatomically complex devices, comprising a custom-made ovine tibia scaffold and a bioreactor chamber in one single step.

\section{Materials and methods}

\subsection{Design and fabrication concept}

The general design methodology employed in this study begins with the generation of a 3D numerical model for any tissue or organ by micro-computed tomography (micro-CT) imaging which is then converted into a 3D replica with pre-determined porosity. An outer shell, referred to hereafter as the bioreactor chamber, with inlets/outlets is then designed around the replica in order to manufacture, in one step, an anatomically shaped device comprising an anatomically optimized scaffold inserted into a customized bioreactor chamber. This allows for the perfusion of the resulting scaffold during in vitro culture, with better control of the flow and shear stress in the system. As a proof of concept, we scanned a $3 \mathrm{~cm}$ ovine tibia section by micro-CT to create a numerical 3D model which was subsequently imported into 3Ds Max (Autodesk, USA) software. The 3D model was vertically re-oriented and both extremities of the tibial model were trimmed to obtain a 3D model with a total length of $2.5 \mathrm{~cm}$ with horizontally flat top and bottom surfaces (figure 1(b5)) and to facilitate the subsequent fluid flow modelling and fabrication steps. However, the other anatomical features of the tibia were accurately reproduced in the resulting scaffold. The 3D design tool, extrusion, was then used to generate a shell wall around the lateral and top outer surfaces of the 3D model (figure 1(b6)). The generated wall was $1 \mathrm{~mm}$ thick and spaced $1 \mathrm{~mm}$ from the 3D scaffold model's outer surfaces. The top part of the shell was converted into a tubular conical shape with a slope angle of $55^{\circ}$ in order to be self-supporting. This part of the shell was designed to enable adequate fluid 


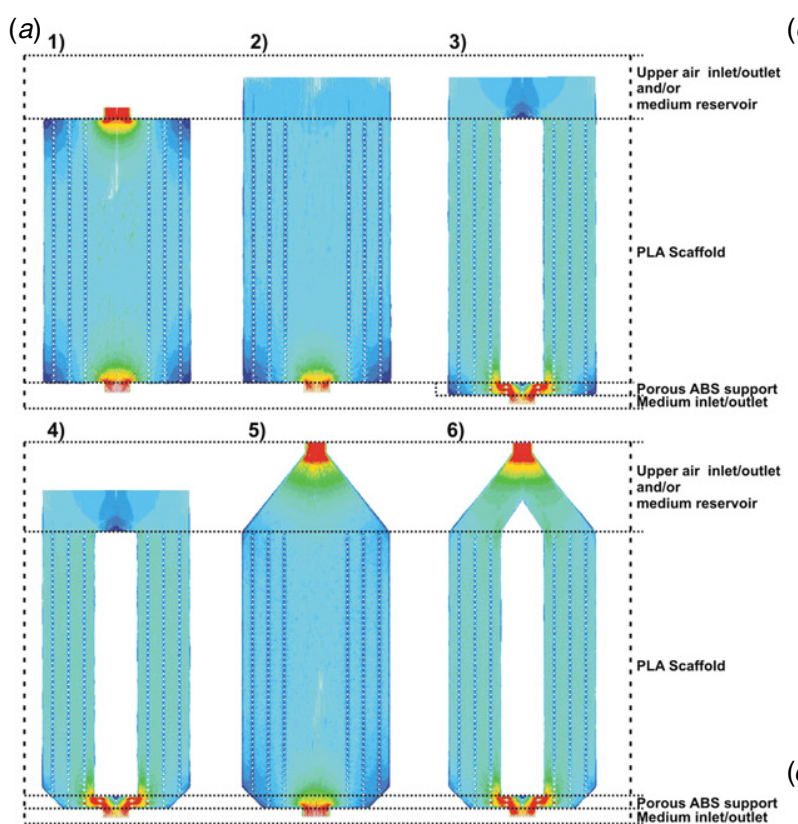

(c)
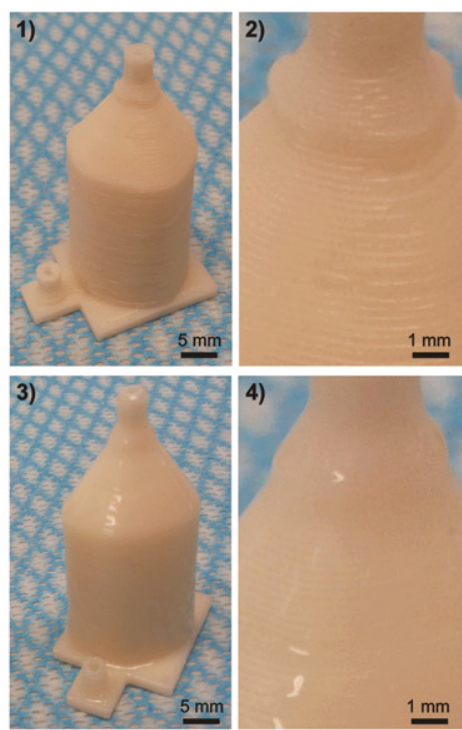

(d)
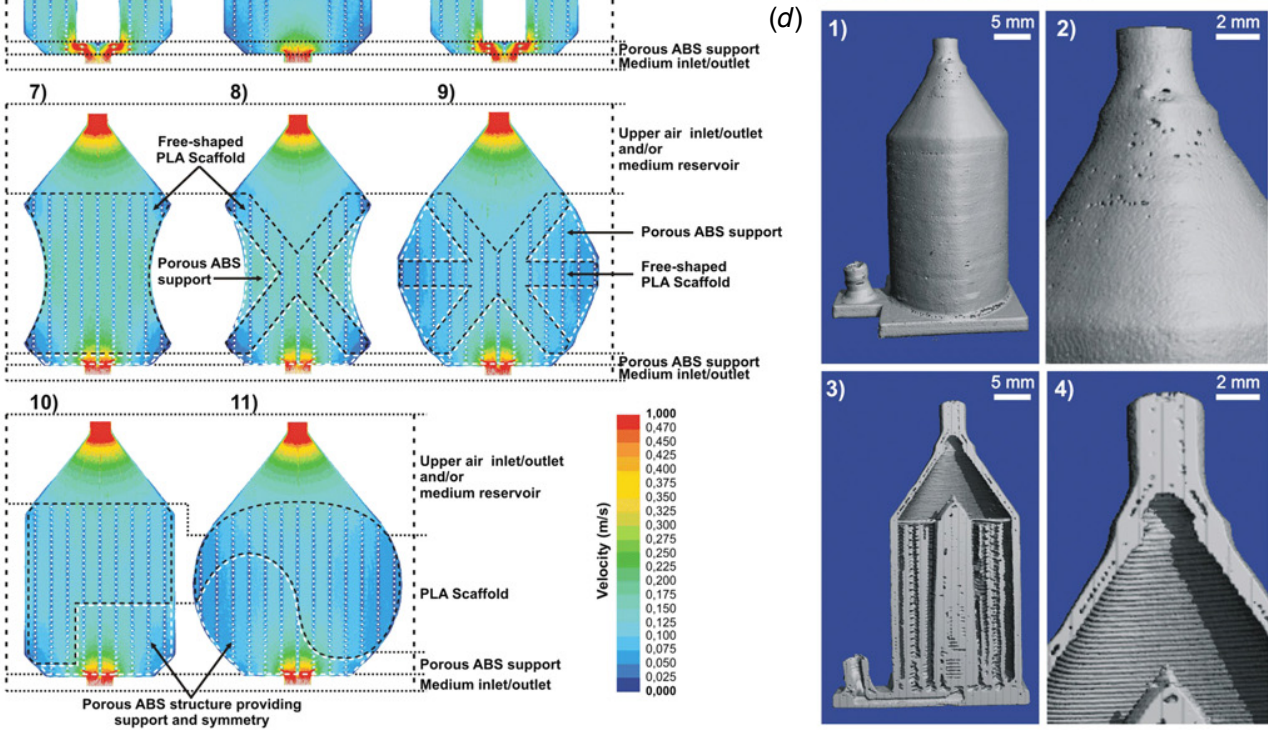

(b)
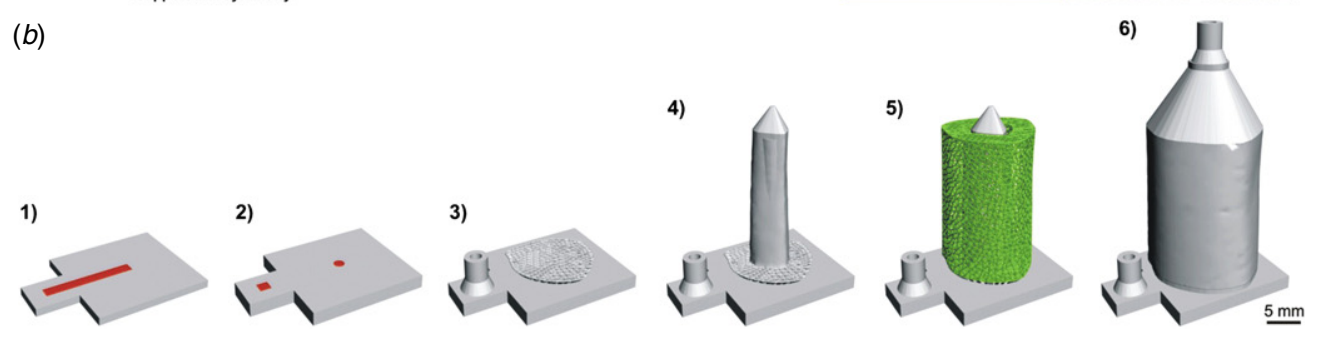

Figure 1. (a) Fluid flow modelling of various tibia-shaped $(a 1)-(a 6)$ and random $(a 7)-(a 11)$ device inner architectures. (a1) A rectangular device with narrow top outlet. (a2) A rectangular device with wide top outlet. (a3) A rectangular device with a support structure (located at the bottom) and a filler column centrally positioned with regard to the scaffold. (a4) A $2 \mathrm{~mm}$-chamfered chamber with support structure, filler column and wide outlet. (a5) A $2 \mathrm{~mm}$ chamfered chamber with support structure and conical outlet but without a filler column. (a6) A $2 \mathrm{~mm}$ chamfered chamber with support structure, filler column and conical outlet, (a7) a device with smooth lateral concavity, (a8) a device with smooth lateral concavity complemented with fluid-homogenizing ABS supports, (a9) a device with a smooth convex shape possessing inner lower and lateral supports, (a10) and (a11) devices adjusted to be asymmetrical employing fluid-homogenizing ABS supports.

(b) Elements of the device to be prototyped. (b1) The base of the device consisting in a lower plate designed with a mini-channel for supplying the culture medium (red) which is covered by another plate containing two holes. (b2), (b3) The medium inlet/outlet and porous structure positioned over the holes. (b4) The filler column centrally positioned with regard to the scaffold. (b5) The tailor-made porous scaffold (green) positioned around the filler column. (b6) The bioreactor chamber surrounding the scaffold. (c) Fabrication and post-processing of the device. $(c 1),(c 2)$ The device after rapid-prototyping. (c3), (c4) The device after treatment with ABS/acetone solution. (d) A micro-CT reconstruction of device. $(d 1),(d 2)$ A reconstruction depicting the smooth outer ABS-made surface of the device which was treated with ABS/acetone solution. (d3) A cross-section depicting the structure of the device containing a NaOH-treated PLA scaffold and a central ABS filler column. (d4) The morphology of the bioreactor chamber which is characteristic of layer-by-layer deposition suggesting that the ABS-acetone post-treatment did not affect the inner wall of the bioreactor chamber (in contrast to the outer wall shown in $(d 2)$ ). 
flow in the interior of the device and to provide sufficient space to contain extra culture medium. The top end of this conical medium reservoir had a slope angle of $90^{\circ}$ creating an inlet/outlet structure with an inner diameter of $2 \mathrm{~mm}$ onto which tubing could be connected. The bottom corners of the chamber were additionally chamfered by $2 \mathrm{~mm}$ with a slope angle of $45^{\circ}$ to improve the hydrodynamic design. On the bottom of the device, a plate containing a $1 \mathrm{~mm}$ wide mini channel was designed (figures $1(b 1)$ and $(b 2)$ ), allowing connectivity of the centre of the plate to a lateral $2 \mathrm{~mm}$ diameter inlet/outlet (figure 1(b3)). Furthermore, by using the same extrusion design tool, a plain column was also generated to fill the empty space located in the centre of the tibia-shaped model (figure 1(b4)).

\subsection{Fluid flow modelling}

A computational fluid dynamics approach was used to simulate the flow pattern of the media in various device architectures. The studied architectures diverged in three main aspects: utilization of rectangular/chamfered chamber bottom corners, the presence/absence of a central filler column for filling the void space inside the tibia model and utilization of wide/narrow top inlets/outlets (figures $1(a 1)-(a 6)$ ). Given the high complexity of the device, a simplified model consisting of two-dimensional sections was created in Design Modeler 13.0 software (Ansys, USA). A profile corresponding to the section of a tubular scaffold with a length of $2.5 \mathrm{~cm}$, an outer diameter of $13 \mathrm{~mm}$ and inner diameter of $6 \mathrm{~mm}$ was considered. The thickness of the simplified section was $0.1 \mathrm{~mm}$ while a converged free mesh was generated using automatic element selection. Mesh independence and sufficient shape accuracy could be reached at a maximum face size of $10 \mathrm{~mm}$ by applying a free mesh. A maximum face size of $0.2 \mathrm{~mm}$ was utilized in order to obtain a highly detailed and accurate solution. The fluid flow velocity profiles were calculated using Fluent 13.0 software, within the Ansys Workbench 13.0 platform (Ansys, USA). The pressure at the device's outlet was assumed to be zero and the scaffold and bioreactor chamber were considered to be rigid and impermeable. We assumed that the viscosity and density of the culture medium at $37{ }^{\circ} \mathrm{C}$ were $\eta=1.45 \times 10^{-3} \mathrm{~Pa} \mathrm{~s}$ and $\rho=1000 \mathrm{~kg} \mathrm{~m}^{-3}$ respectively.

To further demonstrate the versatility of our design strategy, several scaffolds with distinct shapes and dimensions were numerically tested with particular attention on the homogeneity of the fluid flow profile (figures $1(a 7)-(a 11)$ ).

\subsection{Conversion, slicing and prototyping of $3 D$ models}

Fluid flow modelling permitted the selection of an optimized device design which was subsequently utilized in the rest of the study. The device's 3D model was then sliced and converted into a G-Code file. The volume corresponding to the $3 \mathrm{D}$ tibia to be converted into a porous scaffold was processed using the open source software Reprap (Online Reprap Community). The 3D tibia model was sliced into $0.27 \mathrm{~mm}$ layers composed of deposition path lines with a spacing of $1.5 \mathrm{~mm}$. The volume corresponding to the outer shell device was sliced and converted to G-Code files using the open source software ReplicatorG (Online ReplicatorG Community). The conversion was performed using two shells, a slice thickness of $0.27 \mathrm{~mm}$, an object infill of $100 \%$, a feed rate of $20 \mathrm{~mm} \mathrm{~s}^{-1}$ and a travel feed rate of $55 \mathrm{~mm}$ $\mathrm{s}^{-1}$ as parameters. The G-Code generated through RepRap and ReplicatorG were then merged in order to generate a single G-Code file.

The prototyping of the devices was performed using an open source dual extrusion rapid prototyping machine (Replicator, Makerbot Industries, USA). The materials used were poly(lactic) acid (PLA) Ingeo 4043D (NatureWorks LLC, USA) for the porous scaffolds and acrylonitrile butadiene styrene (ABS) (Makerbot industries, USA) for the bioreactor chamber. PLA is a commonly utilized and well-accepted material for various biomedical applications [19]. PLLA has very good mechanical properties with a high yield stress and is therefore a good candidate for high-load bearing bone tissue engineering applications. ABS is widely utilized in industrial applications, possesses the advantage of being inert and thus is suitable for the fabrication of non-implantable devices (in this case the bioreactor chambers). The temperature used for fusing both materials in their corresponding nozzles was $220^{\circ} \mathrm{C}$. Both materials were deposited through coordinated and alternating operation of the nozzles.

\subsection{Bioreactor surface treatment}

In order to ensure that fluid leakage could not occur during subsequent cell culture, a post-treatment coating of ABS was applied to the outer bioreactor wall surfaces to seal any porosity. In brief, after capping all inlets/outlets, the devices were immersed for $2 \mathrm{~s}$ in an ABS/acetone $60 \mathrm{mg} \mathrm{mL}^{-1}$ solution and then air-dried at room temperature for $30 \mathrm{~min}$. This procedure was performed twice. Finally, the devices were washed several times in distilled water to remove residual solvent.

\subsection{Scaffold surface treatment}

Given the hydrophobic nature of PLA, which could hinder appropriate and homogeneous fluid perfusion through the device, alkaline etching was performed using a $2 \mathrm{M}$ sodium hydroxide $(\mathrm{NaOH})$ solution. The devices were rinsed in $100 \%$ ethanol solution under vacuum for $1 \mathrm{~h}$ to pre-wet the scaffold fibre surfaces. After the removal of the ethanol, sodium hydroxide solution was perfused through the device until the bioreactor was completely filled. The devices were incubated for $30 \mathrm{~min}$ at room temperature under vacuum prior to a secondary incubation at $37^{\circ} \mathrm{C}$ for $60 \mathrm{~min}$. Finally, the devices were washed several times with distilled water and then airdried. In order to assess the efficacy of the $\mathrm{NaOH}$ treatment, the same protocol was performed on $8 \mathrm{~mm}$ cubic scaffolds with similar architecture but not inserted in the device. Images of fluid infiltration within the scaffolds prior to and after $\mathrm{NaOH}$ treatment were taken using a digital camera. The alkaline etching ( $\mathrm{NaOH}$ treatment) was utilized to increase the hydrophilicity of the scaffold surface. Base or acid treatments have been shown to increase the surface density of carboxylic acid groups of aliphatic polyester. This is generally associated 
with an increase in the surface roughness and enhanced cell attachment [20, 21]. However, the device developed in this study enables any type of surface modification as long as it is performed in an aqueous environment. We have demonstrated in a previous work that a calcium phosphate coating can be performed on scaffolds resulting in enhanced alkaline phosphatase activity, mineralization and bone formation in osteoblast-seeded scaffolds [22].

\subsection{Micro-computerized tomography analysis (micro-CT)}

The devices were analyzed by micro-CT ( $\mu$ CT40, SCANCO Medical AG, Brüttisellen, Switzerland) at a resolution of $12 \mu \mathrm{m}$, a voltage of $55 \mathrm{kVp}$ and a current of $175 \mu \mathrm{A}$. 3D images were reconstructed from the scans by the micro-CT system software.

\subsection{In vitro study}

Cell culture took place over six weeks and was performed under bi-directional perfusion. A $1 \mathrm{~mL}$ sterile syringe was connected to the device's medium inlet using sterile silicone tubing (figures $3(a 1)$ and $(a 2)$ ). This apparatus was mounted on a multi-syringe adapter placed on an Aladdin syringe pump (World Precision Instruments, USA). A total of six devices (each with a dedicated syringe) were simultaneously cultured in this system. The devices were first sterilized by perfusion with $50 \%$ ethanol solution for $20 \mathrm{~min}$ and dried in a sterile biohazard safety cabinet for $2 \mathrm{~h}$. The devices were manually filled with $4 \mathrm{~mL}$ of basal medium and bi-directionally perfused overnight at a flow rate of $0.4 \mathrm{~mL} \mathrm{~min}^{-1}$ with flow direction inversion every $100 \mathrm{~s}$ using the syringe system. This hydration step aimed to promote the pre-adsorption of proteins to the scaffold surfaces and hence facilitate subsequent cell attachment. In order to ensure the sterility of the device during the culture period while still allowing gas exchange with the incubator's environment, a $0.2 \mu \mathrm{m}$ pore size filter $(13 \mathrm{~mm}$ diameter) (Pall, USA) was connected to the outlet positioned at the top of the device.

Primary human osteoblasts were collected from a patient undergoing hip surgery. Ethics approval for the use of human osteoblasts in this experiment was granted from The Prince Charles Hospital ethics committee (ethics clearance number: EC2310) and Queensland University of Technology ethics committee (ethics clearance number 0600000232). The osteoblasts were expanded in basal alpha MEM modified medium (Gibco, USA) supplemented with $10 \%$ foetal calf serum and $1 \%$ penicillin/streptomycin and used at passage 2 . When reaching confluence, the cells were trypsinized and aliquots of $1 \times 10^{6}$ osteoblasts in $250 \mu$ l of media were prepared. The medium previously contained in each device was then mixed with a cell suspension aliquot and perfused back into the device through the silicone tube. The devices were bi-directionally perfused for $24 \mathrm{~h}$ at a flow rate of $0.4 \mathrm{~mL} \mathrm{~min}^{-1}$ in order to allow cell attachment. The perfusion direction was automatically inverted every $100 \mathrm{~s}$ utilizing an external microcontroller connected to the syringe pump.

After $24 \mathrm{~h}$, the culture medium was replaced by fresh medium and the flow rate was increased to $0.8 \mathrm{~mL} \mathrm{~min}^{-1}$ whereas the flow inversion frequency was reduced to $50 \mathrm{~s}$. The medium was changed weekly and the scaffolds were collected for analysis at two and six weeks postseeding. The samples were analysed after two weeks of culture as a low amount of cells was initially seeded in order to test the device's ability to support cell proliferation. The six weeks time point was chosen in order to assess the system's ability to maintain sterility and cell viability over a prolonged period of time. The scaffold collection was performed as follows: the devices were detached from the syringe pump by removing the silicone tubings and placed in a sterile biohazard safety cabinet. The scaffolds were retrieved from the interior of the bioreactor chamber by manually sectioning the bioreactor walls using sterile tweezers and scissors. The collected scaffolds were processed for either 3(4,5-dimethylthiazol-2-yl)-2,5-diphenyltetrazolium bromide (MTT) staining, live/dead assay, confocal laser scanning or electron microscopy.

\subsection{Metabolic activity staining}

Metabolically active cells were visualized by using a $1 \mathrm{mg} \mathrm{mL}$ 3-(4,5-dimethylthiazol-2-yl)-2,5-diphenyltetrazolium bromide (MTT) solution. Following retrieval, the scaffolds were washed in phosphate buffered saline (PBS) and then immersed into MTT solution for $30 \mathrm{~min}$. Images were captured by a digital camera mounted on an Eclipse TS100 microscope (Nikon, Tokyo, Japan).

\subsection{Live/dead assay (FDA/PI)}

Cell viability was assessed using a live/dead assay. The scaffolds were washed twice in PBS, incubated for $5 \mathrm{~min}$ at $37{ }^{\circ} \mathrm{C}$ in PBS containing $0.67 \mu \mathrm{g} \mathrm{mL}-1$ fluorescein diacetate (FDA) and $5 \mu \mathrm{g} \mathrm{mL} \mathrm{mL}^{-1}$ propidium iodide (PI) (both Invitrogen) and washed again in PBS. The cellularized scaffolds were analysed using a confocal laser scanning microscope (Leica TCS SP5, Leica Microsystems, GmbH). The excitation/emission wavelengths used for imaging the FDA and PI stainings were respectively 488/518-568 and $561 / 598-795 \mathrm{~nm}$. A semi-quantitative analysis of the cellular viability was performed using ImageJ software to quantify living cells (green) and dead cells (red). A total of seven images from multiple regions of interest of the constructs were captured and analysed for each time point.

\subsection{Scanning electron microscopy}

Osteoblast morphology and distribution in the scaffold was assessed at two and six weeks post-seeding by scanning electron microscopy (SEM). In brief, samples were fixed in $3 \%$ glutaraldehyde, washed in $0.1 \mathrm{M}$ cacodylate buffer and post-fixed in $1 \%$ osmium tetroxide in cacodylate buffer for $1 \mathrm{~h}$, prior to dehydration through sequential graded series of ethanol concentrations. Finally, samples were immersed in hexamethyldisilazane for $60 \mathrm{~min}$, air-dried, mounted onto aluminium stubs and gold coated. Samples were observed on a FEI Quanta 200 Environmental SEM operating at $10 \mathrm{kV}$. Scaffolds without cells were gold coated prior to imaging. 


\section{Results and discussion}

The objective of this study was to develop and assess an innovative biofabrication-rooted technology platform for simultaneously generating anatomically shaped scaffolds and bioreactor chambers. Additive manufacturing is commonly used for fabricating scaffolds for tissue engineering, however it is rarely utilized for manufacturing functional bioreactors. This study is, to our knowledge, the first report on the utilization of rapid prototyping for designing anatomically shaped bioreactors and scaffolds in one manufacturing step. The versatility provided by additive manufacturing technologies enables the design and fabrication of highly complex scaffolds which raise a realm of new possibilities, yet also new challenges strongly related to the in vitro culture methods. Indeed, seeding methods are yet to be developed for homogenously distributing the cells through large volume scaffolds such as the ones utilized in this study. Also, an adequate supply of nutrients and oxygen throughout the entire construct is another parameter of importance for the generation of viable tissue substitutes. Unlike small and regular-shaped scaffolds, the seeding and culture of customized and/or patient-specific scaffolds of large size and volume requires a greater degree of complexity in their design for achieving appropriate in vitro tissue development. In the context of personalized regenerative strategies, highly versatile manufacturing platforms are required for fabrication. Hence, the methodology developed in this work intends to circumvent the above-mentioned challenges while still being cost effective.

\subsection{Fluid flow modelling}

Control over the fluid pattern within perfused scaffolds is essential to achieve effective in vitro culture conditions. Homogenous and laminar flow patterns are among the main features defining an optimal culture system. Therefore, multiple culture chamber designs fitting the outer shape of the tibia section model were tested in order to define the most appropriate design by varying several parameters such as the utilization of rectangular or chamfered chamber bottom corners, the presence or absence of an inner filler column and wide (same diameter as the scaffold) or narrow top (2 mm diameter) inlet/outlet. Figure 1( $a$ ) depicts the velocity profile of the media perfused in these devices as obtained by numerical modelling. It was observed that chamfered geometries were the most efficient in avoiding low velocity sites, in contrast to rectangular geometries where fluid velocities in the vicinity of the rectangular lower corners dropped to under $2.5 \%$ of the inlet fluid velocity. Hidalgo-Bastida et al utilized computational fluid modelling for predicting and comparing fluid patterns into rectangular or circular bioreactor chambers. It was shown that a circular geometry was the most suitable for avoiding both the formation of stagnant zones in the peripheral regions of the scaffolds and high fluid velocity near the chamber's inlets/outlets [23]. However, the regions located between the scaffold and the chamber's inlets/outlets were not taken into account despite potential media accumulation in these areas. Figures $1(a 1)-(a 3)$ clearly show that rectangular bottom corners are prone to the formation of stagnant regions and this is of particular relevance for vertically oriented perfusion chambers and can induce cell apoptosis caused by a lack of nutrients and oxygen. However, this is eliminated to a large extent by the optimized geometry of the bioreactor chamber (figures 1(a4)-(a6)) where fluid velocities were always kept $2.5 \%$ above the initial fluid velocity. The design was further optimized by adding an ABS porous support structure at the base of the bioreactor (figures 1(a3)-(a6)), which proved highly efficient at placing the scaffold away from both stagnant and high fluid velocity regions.

The design of a column internal to the scaffold's void central part allowed efficient steering of the media towards the central region of the scaffold (figures 1(a3), (a4) and (a6)). This also contributed to an increase in the fluid velocity in the scaffold to $15-20 \%$ of the initial value and resulted in a more homogeneous fluid velocity being established. This is in contrast to the design without the ABS column where the fluid velocity was much lower (5-7.5\%) and highly heterogeneous (figures $1(a 5)$ and (a6)). Taking into consideration that the initial flow rate was $0.8 \mathrm{~mL} \mathrm{~min}^{-1}$, this column design resulted in an homogeneous flow rate of $0.12-0.16 \mathrm{~mL} \mathrm{~min}^{-1}$ in the scaffold, which is similar to what was found in previous studies where flow rates within this range positively influenced cellular proliferation and development while at the same time avoiding cell detachment caused by high shear stress [12, 24].

The utilization of a wide or narrow inlet/outlet configuration at the top of the device did not significantly affect the flow pattern in the device, however the narrow outlet configuration (figure 1(a6)) was selected for the subsequent in vitro culture, as it would effectively reduce the risk of contamination by permitting the attachment of a sterile air filter.

We further demonstrated the versatility of the design strategy by testing more complex and technically challenging sizes, shapes and volumes (figures 1(a7)-(a11)). We were able to show that the outer shape of the bioreactor chamber accurately followed the geometrical features of the scaffold to a certain degree of concavity without significantly affecting fluid velocity homogeneity (figure $1(a 7)$ ). When the degree of concavity was too high, a porous ABS support was introduced in the lower and lateral part of the device in order to provide structural support and ensure fluid velocity homogeneity (figures 1(a8) and (a9)). The introduction of porous ABS supports also proved to be effective in enabling fluid flow homogeneity in cases of asymmetrical scaffold profile designs (figures 1(a10) and (a11)).

\subsection{Design, fabrication and post-processing of the device}

Taking into consideration the results obtained from the fluid flow modelling and considering the specific needs for the development of a perfusion bioreactor, an optimized device design was achieved by 3D modelling in the 3Ds Max Software ((figure $1(b))$. The bottom part of the device consisted of a base plate into which a mini channel was integrated (figures $1(b 1)$ and (b2)) to supply sufficient culture medium into the device. A tubular structure was added at one end of the channel to connect the chamber to the culture medium 

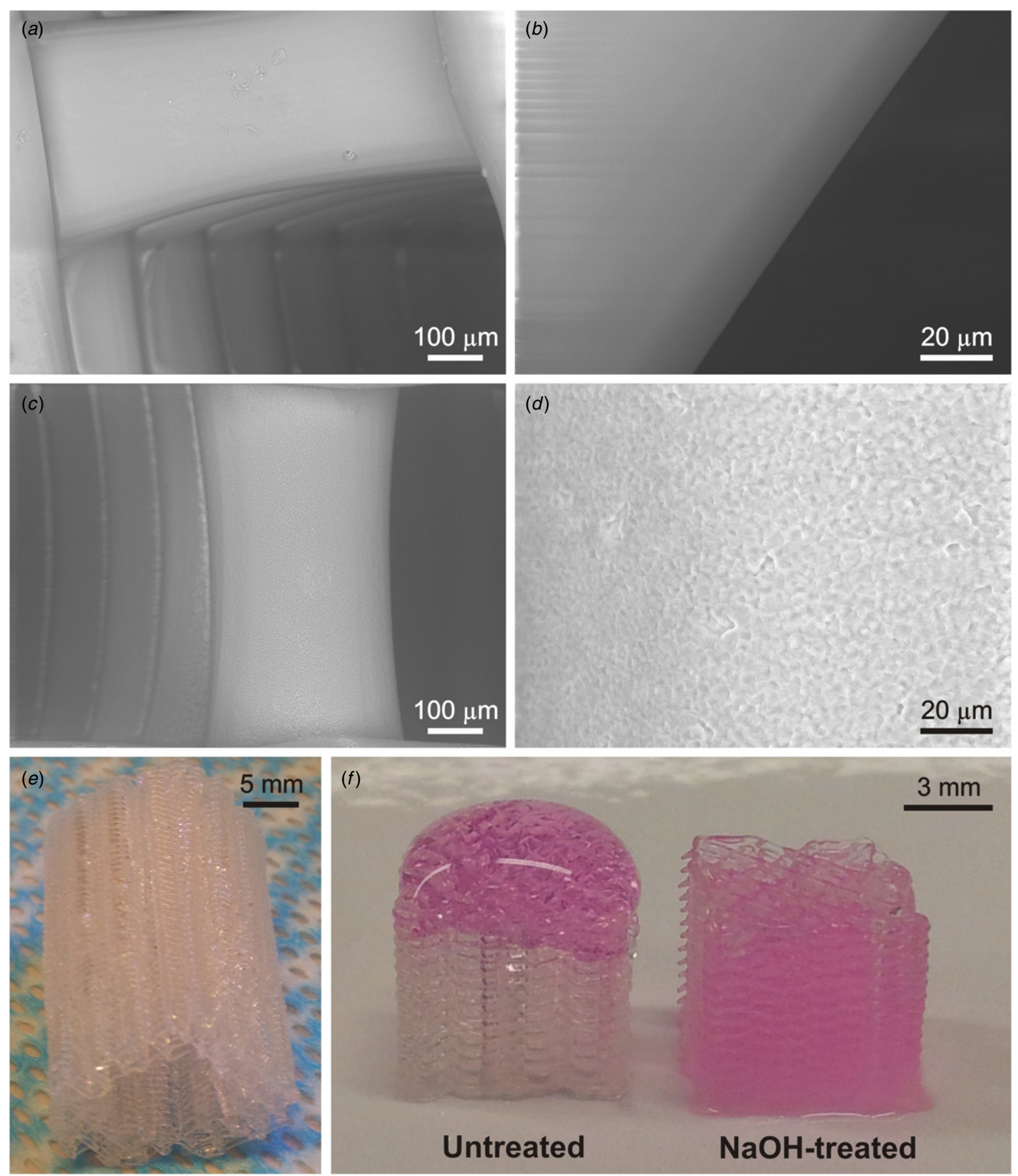

Figure 2. Effect of $\mathrm{NaOH}$ treatment. $(a)$ and $(b)$ Scanning electron micrographs obtained from the surface of untreated, $(a)$ and $(b)$ and $\mathrm{NaOH}$-treated scaffold $(c)$ and $(d)$. $(e)$ Scaffold retrieved from the device. $(f)$ Increased hydrophilicity resulting from the $\mathrm{NaOH}$ treatment, untreated (left) and $\mathrm{NaOH}$-treated test scaffolds (right) when hydrated with culture medium.

reservoir. As already described in the previous section, a porous ABS structure was placed at the bottom of the device to allow homogenous fluid distribution (figure 1(b3)). This porous support was $1 \mathrm{~mm}$ thick and possessed a cross-section similar to that found in the anatomically shaped scaffold. Figure 1(b4) shows the filler column utilized to steer the medium towards the central portions of the scaffold and reduce the volume of medium necessary for cell seeding and subsequent culture. A $1 \mathrm{~mm}$ spacing was maintained between the column and the scaffold to permit fluid circulation in these locations (figure 1(b5)). Finally, an outer bioreactor chamber was designed around the scaffold and its porous support (figure 1(b6)). This chamber was positioned $1 \mathrm{~mm}$ from the scaffold and matched the anatomical shape of the scaffold. While further reducing the volume of medium utilized during the in vitro culture, this also allowed greater control over the fluid flow pattern. Indeed, a bioreactor chamber that accurately follows the shape of the scaffold ensures effective circulation of the culture medium through the interior of the construct, not only around its periphery. As seen in the fluid flow simulations, the hydrodynamic design provided by both the inner column and the bioreactor chamber resulted in homogenous fluid patterns throughout the interior of the scaffold. Commercially available bioreactor culture systems 
(a)
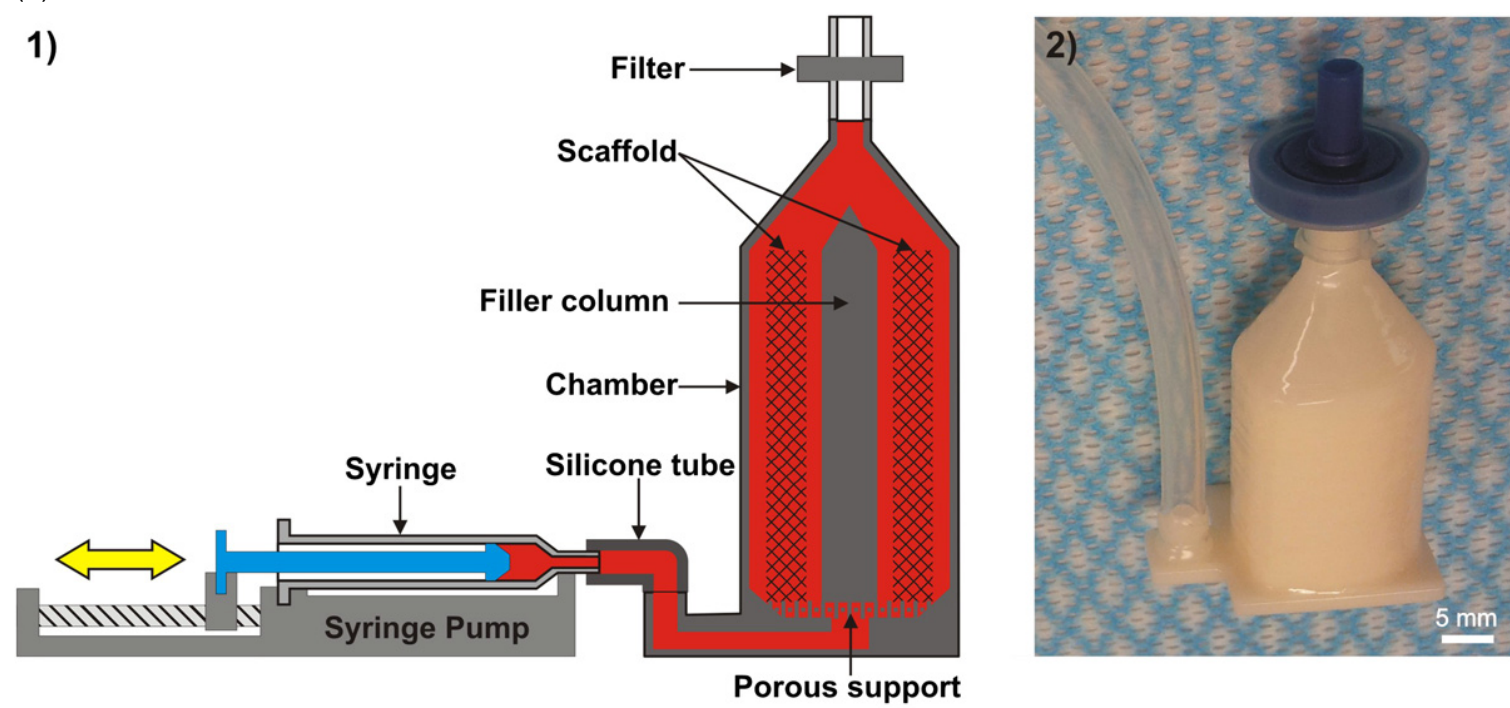

(b)
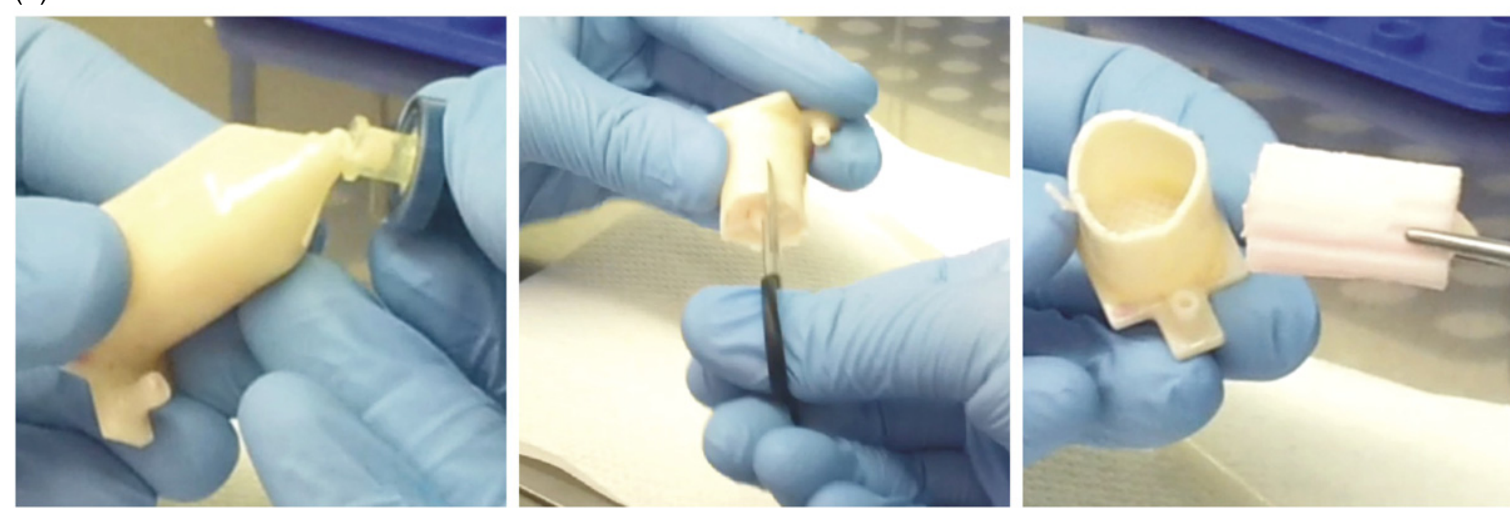

Figure 3. (a) The structure of the cell culture apparatus. (a1) A schematic representation of the device being bi-directionally perfused with culture medium using a syringe mounted onto a syringe pump. (a2) The device with the upper inlet capped with a syringe filter to preserve sterility and for gas exchange. A silicone tube is connected to the lower inlet/outlet for bi-directional circulation of the culture medium.

(b) The procedure for retrieving the construct from the culture device by manually breaking and cutting with the aid of sterile scissors and tweezers.

such as the U-Cup (Cellec Biotek AG, Switzerland) have shown that bi-directional perfusion enhances cell seeding efficiency and distribution and results in more reproducible bone formation $[6,25]$. In contrast to our device, the U-Cup is only capable of culturing small cylindrical constructs and hence lacks the versatility required for tailor-made applications in which the construct's shape and architecture need to be optimized on a case-to-case basis.

The final design (figure 1(b6)) was saved as an STL file, converted to G-Code using RepRap and ReplicatorG softwares and finally prototyped by the Makerbot. The fabrication time for each device was $45 \mathrm{~min}$. As some open porosity remained within the bioreactor chamber, a post-treatment with an ABS solution was utilized in order to seal these pores ensuring that no leakage would occur during the subsequent cell culture. Acetone-based solutions are commonly used as cements for repairing, welding and smoothing, as well as for waterproofing structures made of ABS [26, 27]. As shown in figure 1(d), the treatment reduced the outer surface roughness of the bioreactor chamber and all interlayer micro-gaps were eliminated. This significantly affected the appearance of the chamber's outer wall as shown in figure $1(c)$ as the post-treated material appeared smoother with a glossy finish.

The $\mathrm{NaOH}$ post-treatment on the PLA scaffold resulted in high surface roughness and consequently higher hydrophilicity when compared to untreated samples (figure 2), which was in accordance with a previous study [28]. This post-treatment exemplifies the fact that other types of surface modification can be performed on the scaffold by simply perfusing fluids within the device such as simulated body fluid for a biomimetic coating [29-31].

The high versatility provided by additive manufacturing, combined with the concept we have developed in this study, permits and facilitates the optimization of numerous devices with different shapes and sizes where various design features such as additional inlets/outlets can be incorporated. Indeed, unlike traditional bioreactors, one major advantage of this manufacturing strategy is that both culture chamber and scaffold designs can be freely modified and optimized according to the intended tissue engineering application without any additional engineering steps or increased costs. 
(a)

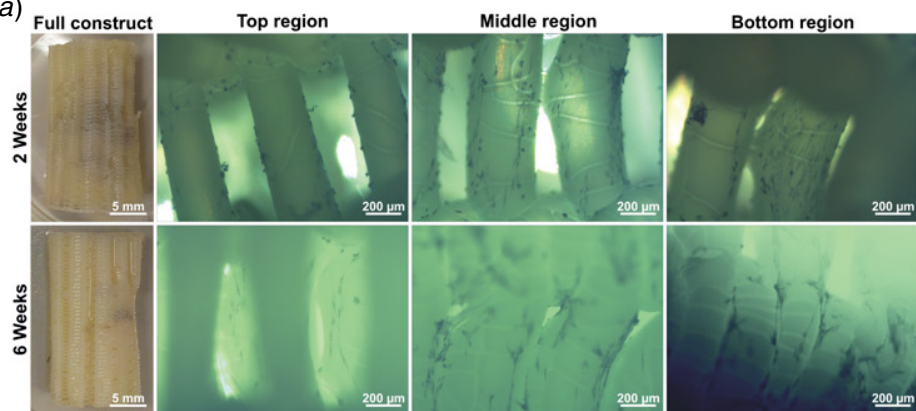

(c)
Top region

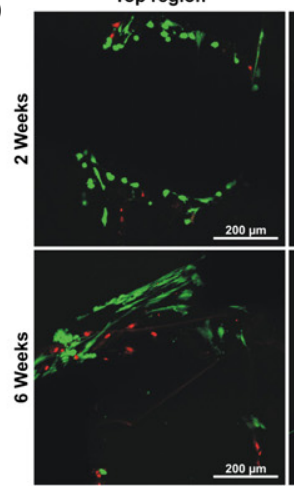
Middle region

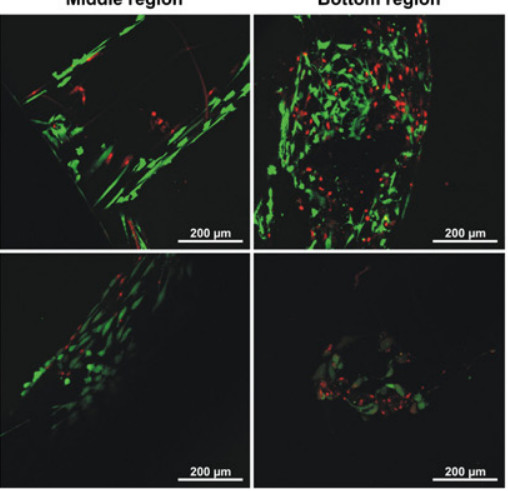

(b)

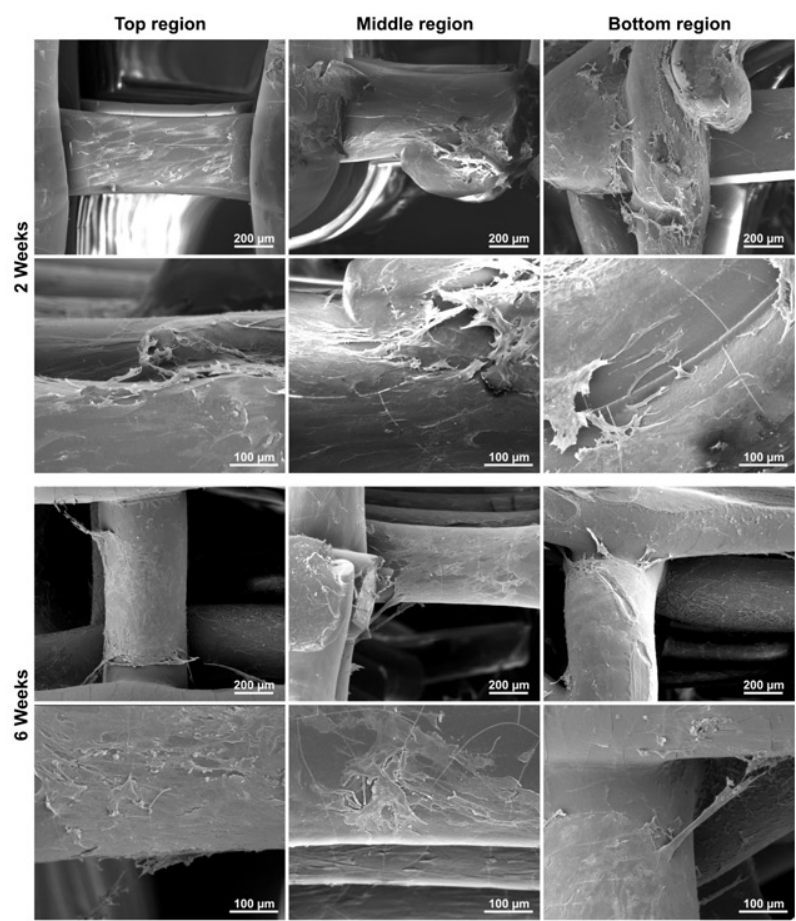

Figure 4. (a) MTT staining of human osteoblast-cultured scaffolds after two and six week culture times. (b) Scanning electron micrographs of human osteoblast-cultured scaffolds after two and six week culture times. (c) FDA/PI viability staining of human osteoblast-cultured scaffolds after two and six week culture times showing viable cells in green and dead cells in red.

In this proof-of-principle study, a scaffold design for a well-established large preclinical animal model was chosen, namely for regenerating a large segmental defect of a sheep [32]. Scaffolds with more complex shapes and heterogeneous architectures could be fabricated using the method described in this study (see supplementary figure 1 (available from stacks.iop.org/BF/6/035006/mmedia)). The mechanical properties of such scaffolds can also be highly tuneable through optimizing the processing parameters. In a study by Ciurana et al [33] it was shown that, by simply varying parameters such as degree of porosity, deposition angle, slenderness and distance between filaments, scaffolds very similar to those employed in this work could reach Young's modulus values as high as 1,2 $\mathrm{GPa}$ which is greater than Young's modulus of cancelous bone $(0.02-0.5 \mathrm{GPa})$ and around $50 \%$ of the lower range of Young's modulus of cortical bone (3-30 GPa) [34]. In order to reach Young's modulus values within the range found in cortical bone, reinforcements such as hydroxyapatite can further be added to PLA generating composite materials with enhanced mechanical properties [35]. The high versatility in the design and manufacturing of both the scaffold and the culture chamber is the main characteristic that distinguishes this technology platform from other current scaffold-based bone tissue engineering strategies. For instance, a comparison with the strategy developed by Grayson et al [11] clearly shows the superior properties of our concept with regard to design and manufacturing. Indeed, the fabrication is performed in a single step while allowing the maintenance of a high degree of accuracy in the architecture and the shape of the scaffold and/or bioreactor chamber.

\subsection{Perfusion cell culture}

The perfusion bioreactor was composed of various devices with their top air inlet/outlets capped with syringe filters (figure 3(a2)) and connected to individual syringes mounted into one single syringe pump by means of a multi-syringe adapter (figure 3(a1)). After an overnight incubation of the scaffold in culture medium, the cell suspension was injected into the device and the bi-directional perfusion was applied to achieve homogeneous seeding. After two and six weeks of culture, the scaffolds were collected by manually opening the devices utilizing sterile scissors and tweezers as depicted in figure $3(b)$. The MTT staining revealed the distribution of viable cells at two and six weeks (figure $4(a)$ ). Indeed cells were well-distributed throughout the length of the scaffold, and also on the outer and inner portions of the architecture confirming that appropriate in vitro culture conditions were achieved. The MTT staining observations were corroborated by SEM analysis (figure 4(b)) which showed homogeneous cell distribution and intimate attachment onto the scaffold. It was also observed that the cells were covering the polymer struts of the scaffold, although this was more pronounced at six weeks post-seeding. In some cases cells were capable of spanning the nearby struts as shown in figure $4(b)$.

FDA/PI staining and confocal laser scanning microscopy confirmed the homogeneity of the cell distribution while assessing their viability (figure 4(c)) despite the technical challenges encountered when imaging samples of larger dimensions via confocal laser microscopy. Therefore, the scaffolds were divided into three zones-top, middle and bottom—similarly to what was performed in the rest of 
Table 1. Statistical analysis of percentage of live cellular content in cultured samples.

\begin{tabular}{lll}
\hline & 2 weeks & 6 weeks \\
\hline Percentage of live cells $(\%)$ & $70.15 \pm 10.14$ & $74.29 \pm 18.45$ \\
\hline
\end{tabular}

this study. This revealed that, despite the presence of some necrotic cells (stained in red), the vast majority of the cells were alive (green). No significant difference in cell viability was observed when semi-quantitatively comparing the various sites of the construct. Although a slight increase in necrotic cells in the bottom region was observed, it did not reach statistical significance. The percentage of living cells at two and six weeks was $70 \% \pm 10$ and $74 \% \pm 18$ respectively (table 1). These results demonstrate that nutrients were able to homogeneously diffuse throughout the entire perfusion device, resulting in an even distribution of viable cells in the scaffold and validating the fluid flow model predicting a homogenous flow pattern inside the device.

\section{Conclusions}

This study presents a unique concept based on additive manufacturing for the integrated and simultaneous manufacturing of anatomically shaped scaffolds and customized bioreactor chambers. We foresee that this innovative concept will represent a significant advance in further implementing the currently available biofabrication toolbox towards the development of automated customization of tissue engineered products.

\section{Acknowledgments}

This work was supported by the NHMRC, the Australian Research Council and Hans Fischer Senior Fellowship, IAS-TUM. Pedro Costa acknowledges the Portuguese Foundation for Science and Technology for his $\mathrm{PhD}$ grant (SFRH/BD/62452/2009).

\section{References}

[1] Bates S 2010 Progress towards personalized medicine Drug Discov. Today 15 115-20

[2] Hutmacher D 2013 A road map for a tissue engineering concept for restoring structure and function after limb loss J. Mater. Sci., Mater. Med. 24 2659-63

[3] Grylls R 2003 Intricate parts from the inside out Mach. Des. 75 56-62

[4] Martin I, Wendt D and Heberer M 2004 The role of bioreactors in tissue engineering Trends Biotechnol. 22 80-86

[5] Bancroft G N, Sikavitsas V I and Mikos A G 2003 Design of a flow perfusion bioreactor system for bone tissueengineering applications Tissue Eng. 9 549-54

[6] Wendt D, Marsano A, Jakob M, Heberer M and Martin I 2003 Oscillating perfusion of cell suspensions through three-dimensional scaffolds enhances cell seeding efficiency and uniformity Biotechnol. Bioeng. 84 205-14

[7] Costa P F, Martins A, Neves N M, Gomes M E and Reis R L 2009 Multichamber bioreactor with bidirectional perfusion integrated in culture system for tissue engineering strategies European Patent Application 09009863

[8] Orr D E and Burg K J L 2008 Design of a modular bioreactor to incorporate both perfusion flow and hydrostatic compression for tissue engineering applications Ann. Biomed. Eng. 36 1228-41

[9] Schulz R M, Wuestneck N, van Donkelaar C C, Shelton J C and Bader A 2008 Development and validation of a novel bioreactor system for load- and perfusion-controlled tissue engineering of chondrocyte-constructs Biotechnol. Bioeng. $101714-28$

[10] Janssen F W, Oostra J, van Oorschot A and van Blitterswijk C A 2006 A perfusion bioreactor system capable of producing clinically relevant volumes of tissue-engineered bone: in vivo bone formation showing proof of concept Biomaterials 27 315-23

[11] Grayson W L, Frohlich M, Yeager K, Bhumiratana S, Chan M E, Cannizzaro C, Wan L Q, Liu X S, Guo X E and Vunjak-Novakovic G 2010 Engineering anatomically shaped human bone grafts Proc. Natl Acad. Sci. USA 107 3299-304

[12] Goncalves A, Costa P, Rodrigues M T, Dias I R, Reis R L and Gomes M E 2011 Effect of flow perfusion conditions in the chondrogenic differentiation of bone marrow stromal cells cultured onto starch based biodegradable scaffolds Acta Biomater. 7 1644-52

[13] Bancroft G N, Sikavitsast V I, van den Dolder J, Sheffield T L, Ambrose C G, Jansen J A and Mikos A G 2002 Fluid flow increases mineralized matrix deposition in $3 \mathrm{D}$ perfusion culture of marrow stromal osteloblasts in a dose-dependent manner Proc. Natl Acad. Sci. USA 99 12600-5

[14] Cartmell S H, Porter B D, Garcia A J and Guldberg R E 2003 Effects of medium perfusion rate on cell-seeded three-dimensional bone constructs in vitro Tissue Eng. 9 1197-203

[15] Goldstein A S, Juarez T M, Helmke C D, Gustin M C and Mikos A G 2001 Effect of convection on osteoblastic cell growth and function in biodegradable polymer foam scaffolds Biomaterials 22 1279-88

[16] Grayson W L, Bhumiratana S, Cannizzaro C, Chao P H G, Lennon D P, Caplan A I and Vunjak-Novakovic G 2008 Effects of initial seeding density and fluid perfusion rate on formation of tissue-engineered bone Tissue Eng A 14 1809-20

[17] Meinel L, Karageorgiou V, Fajardo R, Snyder B, Shinde-Patil V, Zichner L, Kaplan D, Langer R and Vunjak-Novakovic G 2004 Bone tissue engineering using human mesenchymal stem cells: effects of scaffold material and medium flow Ann. Biomed. Eng. 32 112-22

[18] Sikavitsas V I, Bancroft G N, Holtorf H L, Jansen J A and Mikos A G 2003 Mineralized matrix deposition by marrow stromal osteoblasts in 3D perfusion culture increases with increasing fluid shear forces Proc. Natl Acad. Sci. USA 100 14683-8

[19] Vaquette C, Frochot C, Rahouadj R and Wang X 2008 An innovative method to obtain porous PLLA scaffolds with highly spherical and interconnected pores J. Biomed. Mater. Res. B 86B 9-17

[20] Gao J, Niklason L and Langer R 1998 Surface hydrolysis of poly(glycolic acid) meshes increases the seeding density of vascular smooth muscle cells J. Biomed. Mater. Res. 42 417-24

[21] Vaquette C, Fawzi-Grancher S, Lavalle P, Frochot C, Viriot M L, Muller S and Wang X 2006 In vitro biocompatibility of different polyester membranes Biomed. Mater. Eng. 16 S131-6

[22] Costa P F, Vaquette C, Zhang Q, Reis R L, Ivanovski S and Hutmacher D W 2013 Advanced tissue engineering 
scaffold design for regeneration of the complex hierarchical periodontal structure J. Clin. Periodontol. 41 283-94

[23] Hidalgo-Bastida L A, Thirunavukkarasu S, Griffiths S, Cartmell S H and Naire S 2012 Modeling and design of optimal flow perfusion bioreactors for tissue engineering applications Biotechnol. Bioeng. 109 1095-99

[24] Alvarez-Barreto J F, Linehan S M, Shambaugh R L and Sikavitsas V I 2007 Flow perfusion improves seeding of tissue engineering scaffolds with different architectures Ann. Biomed. Eng. 35 429-42

[25] Scherberich A, Galli R, Jaquiery C, Farhadi J and Martin I 2007 Three-dimensional perfusion culture of human adipose tissue-derived endothelial and osteoblastic progenitors generates osteogenic constructs with intrinsic vascularization capacity Stem Cells $251823-9$

[26] Galantucci L M, Lavecchia F and Percoco G 2009 Experimental study aiming to enhance the surface finish of fused deposition modeled parts CIRP Ann. Manuf. Technol. 58 189-92

[27] Galantucci L M, Lavecchia F and Percoco G 2010 Quantitative analysis of a chemical treatment to reduce roughness of parts fabricated using fused deposition modeling CIRP Ann. Manuf. Technol. 59 247-50

[28] Yang J, Wan Y Q, Tu C F, Cai Q, Bei J Z and Wang S G 2003 Enhancing the cell affinity of macroporous poly(L-lactide) cell scaffold by a convenient surface modification method Polym Int. 52 1892-9
[29] Kokubo T, Kushitani H, Sakka S, Kitsugi T and Yamamuro T 1990 Solutions able to reproduce in vivo surface-structure changes in bioactive glass-ceramic a-W3 J. Biomed. Mater. Res. 24 721-34

[30] Kokubo T, Ito S, Huang Z T, Hayashi T, Sakka S, Kitsugi T and Yamamuro T $1990 \mathrm{Ca}$, P-rich layer formed on high-strength bioactive glass-ceramic A-W J. Biomed. Mater. Res. 24 331-43

[31] Vaquette C, Ivanovski S, Hamlet S M and Hutmacher D W 2013 Effect of culture conditions and calcium phosphate coating on ectopic bone formation Biomaterials 34 5538-51

[32] Reichert J C, Wullschleger M E, Cipitria A, Lienau J, Cheng T K, Schutz M A, Duda G N, Noth U, Eulert J and Hutmacher D W 2011 Custom-made composite scaffolds for segmental defect repair in long bones Int. Orthop. 35 1229-36

[33] de Ciurana J, Serenóa L and Vallès È 2013 Selecting process parameters in RepRap additive manufacturing system for PLA scaffolds manufacture Proc. CIRP 5 152-7

[34] Sabir M I, Xu X X and Li L 2009 A review on biodegradable polymeric materials for bone tissue engineering applications J. Mater. Sci. 44 5713-24

[35] Russias J, Saiz E, Nalla R K, Gryn K, Ritchie R O and Tomsia A P 2006 Fabrication and mechanical properties of PLA/HA composites: a study of in vitro degradation Mater. Sci. Eng. C 26 1289-95 